\title{
Meditation-induced changes in high-frequency heart rate variability predict smoking outcomes
}

\author{
Daniel J. Libby ${ }^{1,2,3}$, Patrick D. Worhunsky ${ }^{1}$, Corey E. Pilver ${ }^{1,2}$ and Judson A. Brewer ${ }^{1,2 *}$ \\ Department of Psychiatry, Yale University School of Medicine, New Haven, CT, USA \\ 2 VA Connecticut Healthcare System, West Haven, CT, USA \\ ${ }^{3}$ New England Mental IIIness Research Education and Clinical Centers, Connecticut VA Health Care System, West Haven, CT, USA
}

\section{Edited by:}

Amishi P. Jha, University of

Miami, USA

Reviewed by:

J. David Creswell, Carnegie Mellon

University, USA

Charles Raison, University of

Arizona, USA

\section{*Correspondence:}

Judson A. Brewer, VA Connecticut Healthcare System, 950 Campbell Ave., Building 36, Room 142, West Haven, CT 06516, USA.

e-mail: judson.brewer@yale.edu

\begin{abstract}
Background: High-frequency heart rate variability (HF-HRV) is a measure of parasympathetic nervous system (PNS) output that has been associated with enhanced self-regulation. Low resting levels of HF-HRV are associated with nicotine dependence and blunted stress-related changes in HF-HRV are associated with decreased ability to resist smoking. Meditation has been shown to increase HF-HRV. However, it is unknown whether tonic levels of HF-HRV or acute changes in HF-HRV during meditation predict treatment responses in addictive behaviors such as smoking cessation. Purpose: To investigate the relationship between HF-HRV and subsequent smoking outcomes. Methods: HF-HRV during resting baseline and during mindfulness meditation was measured within two weeks of completing a 4-week smoking cessation intervention in a sample of 31 community participants. Self-report measures of smoking were obtained at a follow up 17-weeks after the initiation of treatment. Results: Regression analyses indicated that individuals exhibiting acute increases in HF-HRV from resting baseline to meditation smoked fewer cigarettes at follow-up than those who exhibited acute decreases in HF-HRV $(b=-4.89, p=0.008)$. Conclusion: Acute changes in HF-HRV in response to meditation may be a useful tool to predict smoking cessation treatment response.
\end{abstract}

Keywords: heart rate variability, mindfulness, meditation, smoking cessation
The rhythm of a healthy human heart naturally fluctuates. This normal variability in heart rate occurs, in part, from the synergistic actions of the sympathetic and parasympathetic branches of the autonomic nervous system (ANS), which act through various neural and physiological mechanisms to allow the cardiovascular system to adapt and respond appropriately to varying internal and external demands and conditions (McDonald, 1980; Berntson et al., 1993a,b, 1997). Fluctuations that occur in the 0.15 to 0.4 hertz $(\mathrm{Hz})$ frequency range are a result of Respiratory Sinus Arrhythmia (RSA), the increase and decrease in heart rate that occurs with inhalation and exhalation, respectively, and are associated with parasympathetic nervous system (PNS) activity via efferent traffic in the vagus $\left(10^{\text {th }}\right.$ cranial) nerve (Berntson et al., 1993b). High-frequency heart rate variability (HF-HRV) is a measure of the fluctuations in this frequency range and is a reliable measure of PNS activity (Task Force, 1996; Berntson et al., 1997).

Low PNS activity, as reflected in low levels of HF-HRV, is a marker of poor psychological health. Low resting levels of HFHRV measured over a few minutes and low HF-HRV over the course of $24 \mathrm{~h}$, has been associated with nicotine dependence (Gallagher et al., 1992; Kupari et al., 1993), as well as with trait negative affect including hostility, depression, and anxiety (Fuller, 1992; Sloan et al., 1994; Light et al., 1998; Bleil et al.,

Abbreviations: HF-HRV, high-frequency heart rate variability; RSA, respiratory sinus arrhythmia
2008), and a variety of affective and anxiety disorders including major depression (Rechlin et al., 1994; Nahshoni et al., 2004; Carney et al., 2005), bipolar disorder (Cohen et al., 2003), generalized anxiety disorder (Thayer et al., 1996), panic disorder (Friedman and Thayer, 1998) and post-traumatic stress disorder (Cohen et al., 1997). High HF-HRV, on the other hand has been associated with smoking abstinence (Minami et al., 1999), positive emotions and interpersonal connectedness (McCraty et al., 1995; Oveis et al., 2009; Kok and Fredrickson, 2010), and regulated emotional responding (Appelhans and Luecken, 2006). Higher levels of HF-HRV during sleep have also been prospectively linked with response to panic disorder treatment (Craske et al., 2005).

Acute changes in HF-HRV are also associated with psychological conditions. In healthy individuals, acute withdrawal of vagal efferent activity occurs in response to stressful situations (Berntson et al., 1994), worry (Thayer et al., 1996), and induction of negative affect (Lane et al., 2009), resulting in acute increases in heart rate (Berntson et al., 1993b), and acute decreases in HFHRV. Blunted HF-HRV reactivity (smaller acute decreases) in response to stressful events, on the other hand, has been found in individuals with borderline personality disorder (Weinberg et al., 2009), post-traumatic stress disorder (Cohen et al., 1998 \#1001) and depression (Rottenberg et al., 2007), and may reflect deficits in self-regulation (Segerstrom and Nes, 2007; Thayer et al., 2009). This aberrant HF-HRV reactivity may predict 
treatment outcome; for example, depressed persons who exhibited a greater degree of HF-HRV reactivity to a sad film were more likely to recover from depression than those with blunted reactivity (Rottenberg et al., 2005). In addition, greater HF-HRV reactivity in children, in response to stress, has been shown to protect against the development of psychopathology (El-Sheikh, 2001), while blunted reactivity has been shown to predict future behavioral problems (Porges, 1996).

In healthy individuals, acute increases in HF-HRV generally occur in response to positive-emotion induction (McCraty et al., 1995), relaxation exercises (Sakakibara et al., 1994; Sarang and Telles, 2006), and meditation (Lehrer et al., 1999; Takahashi et al., 2005; Ditto et al., 2006; Peressutti et al., 2010). However, studies have shown that there is inter-individual variability in autonomic responses to these manipulations. For example, studies have found that some individuals exhibit acute increases in HF-HRV when practicing mindfulness meditations, while other exhibit acute decreases in HF-HRV (Lehrer et al., 1999; Takahashi et al., 2005; Peressutti et al., 2010). Further, these differences may be associated with risk factors for psychopathology. For example, in one study involving a sample of 22 undergraduates, half of the participants exhibited increases, and half exhibited decreases in HF-HRV during a compassion-imagery procedure; decreases in HF-HRV, compared to increases, were associated with selfcriticism and anxious attachment style in this otherwise healthy sample (Rockliff et al., 2008). There have been no published studies, however, examining whether differences in HF-HRV changes from resting baseline to meditation are prospectively related to clinical outcomes.

The association between HF-HRV and psychological functioning may be a function of the relationship between HF-HRV and self-regulation. HF-HRV may index an individual's ability to exert top-down behavioral control via inhibitory processes (Thayer and Lane, 2000). Accordingly, elevated HF-HRV is associated with greater cognitive performance (Hansen et al., 2004; Thayer et al., 2009), better regulation of affect during daily stress (Fabes and Eisenberg, 1997), better modulation of emotional expression (Demaree et al., 2002), better modulation of defensive reactions (Ruiz-Padial et al., 2003), better attention regulation (Johnsen et al., 2003), effective impulse control (Allen et al., 2000), and behavioral regulation (Segerstrom and Nes, 2007). The association between HF-HRV and self-regulation is also supported by evidence showing that the brain structures that regulate autonomic balance overlap with the networks that serve executive, social, affective, attentional, and motivational behaviors (Thayer and Lane, 2000; Thayer and Brosschot, 2005). Higher tonic levels and context-appropriate changes in HF-HRV, then, may be indicative of enhanced emotional and attention regulation abilities (Appelhans and Luecken, 2006), while reduced HF-HRV across situations may be indicative of a lack of emotional and autonomic flexibility that is necessary for healthy psychological and physiological functioning (Rozanski and Kubzansky, 2005; Friedman, 2007).

Psychological interventions that improve emotion regulation and attention regulation may result in improved ANS functioning. Mindfulness meditation, for example, is associated with improved cognitive flexibility (Moore and Malinowski,
2009), reduced affective reactivity and greater emotion regulation (Broderick, 2005; Arch and Craske, 2006; Farb et al., 2010; Goldin and Gross, 2010) as well as improved performance on tests of processing speed, working memory, and attention (Zeidan et al., 2010a). Interestingly, research has suggested that individuals can be trained to be mindful in a relatively short timeframe, although there appears to be individual variability therein. A recent study found that novices accumulating an average of just over $6 \mathrm{~h}$ of practice over the course of five weeks exhibited a significant shift in frontal EEG asymmetry toward a pattern associated with positive, approach-oriented emotions (Moyer et al., 2011). Decreases in amygdala activity in response to both positive and negative emotion-inducing stimuli were found in novice meditators with only $20 \mathrm{~min}$ of practice each day for one week (Taylor et al., 2011). Further, three 20-min training sessions over the course of three days resulted in greater improvements in mood and executive functioning than participants in a sham condition (Zeidan et al., 2010a). Other studies have found improvements in mood, cognition, and cardiovascular variables after brief, onetime instructions in mindfulness (Broderick, 2005; Arch and Craske, 2006; Zeidan et al., 2010b).

In the context of addiction, mindfulness meditation is hypothesized to increase one's ability to maintain a non-reactive mindset when confronted with stress and/or cravings (Brewer et al., 2009, 2010). Treatments for substance use disorders (Bowen et al., 2009; Brewer et al., 2009), and particularly smoking cessation (Cropley et al., 2007; Davis et al., 2007; Brewer et al., 2011), have integrated mindfulness meditation with promising results. For example, college students who received brief (11 minutes) mindfulness-based instructions smoked significantly fewer cigarettes one week later, compared to those who did not receive instructions (Bowen and Marlatt, 2009). Additionally, among individuals participating in a mindfulness training for smoking cessation study, the frequency of meditation practice was associated with reduced smoking (Brewer et al., 2011). Further, individuals with substance use disorders who received mindfulness training showed increased parasympathetic output during stress compared to those who received cognitive behavioral therapy (Brewer et al., 2009). Together, these results suggest that the increased HF-HRV that has been observed during meditation may reflect increased self-regulation capacity or replenishment of depleted self-control resources, which may in turn contribute to the positive effects of mindfulness-based interventions in the treatment of substance-use disorders.

Tobacco dependence is associated with impulsiveness and deficits in self-regulation (Sayette, 2004; Flory and Manuck, 2009). Meditation-induced changes in HF-HRV, on the other hand, may be indicative of the capacity to actively modulate reactivity during stressful states such as during cravings for cigarettes (Thayer et al., 2009). Importantly, a recent study demonstrated that blunted HF-HRV reactivity in response to stress predicted decreased ability to resist the urge to smoke, as well as increased reinforcement from smoking (Ashare et al., 2012). While research has attempted to understand how stress-induced changes in HF-HRV are associated with clinical outcomes, there have been no studies examining the effects of changes in HF-HRV in response to health-enhancing strategies such as 
meditation or relaxation. Also, to date, no studies have examined whether changes in HF-HRV during meditation are associated with smoking cessation outcomes. The ability to increase HF-HRV during periods of meditative or relaxed states may hold predictive power beyond that of stress-induced changes in HF-HRV.

In this exploratory study, we assessed whether resting HFHRV or acute HF-HRV changes during mindfulness meditation were associated with smoking outcomes in a combined group of novice meditators and individuals who had received four weeks of mindfulness training for smoking cessation. As previous studies have demonstrated that individuals can learn to meditate with minimal instruction and that inter-individual variability in the ability to meditate exists in individuals who have received meditation instruction, we sought to determine if physiological changes during meditation were more predictive of outcomes than length of training. In other words, we were interested in whether acute changes in HF-HRV during meditation, regardless of length of training, could predict whether individuals would reduce smoking behavior. Consistent with earlier research that higher baseline HF-HRV and HF-HRV flexibility may both be markers of inhibitory behavioral control, we examined four hypotheses: (1) higher baseline levels of HF-HRV will be associated with fewer cigarettes smoked at 17-week follow-up; (2) increases in HF-HRV compared to decreases from resting baseline to meditation will be associated with fewer cigarettes smoked at 17-week follow-up (3) HF-HRV during meditation will be associated with fewer cigarettes smoked at 17-week follow-up; (4) larger increases in HF-HRV from resting baseline to meditation condition will be associated with number of cigarettes smoked at 17-week follow-up.

\section{MATERIALS AND METHODS PARTICIPANTS}

Participants in the current study were a subsample of individuals recruited for a randomized controlled trial (RCT) of smoking cessation treatment. Participants of the larger RCT were recruited through flyers and media advertisements. Eligible participants were 18-60 years of age, smoked 10 or more cigarettes per day, had little or no prior meditation experience, and reported motivation to quit smoking. Exclusion criteria included current psychiatric medication, serious or unstable medical condition in the past six months, or other substance dependence in the past year.

Before randomization into treatment arms of a 4-week RCT that compared Mindfulness Training for Smoking with the American Lung Association's Freedom From Smoking treatment, a subsample of 34 participants (38.6\% of the RCT) agreed to participate in a functional magnetic resonance imaging (fMRI) meditation laboratory session at the end of the treatment phase of the study (fMRI data will be presented elsewhere). Thirty-one participants provided valid data for analyses. Participants in the current study did not significantly differ from non-participants in age, gender, number of cigarettes smoked the week before treatment, or age of smoking initiation (all $p^{\prime}$ s $>0.05$ ). The study was approved by the Yale University and Connecticut Veterans Affairs Healthcare System institutional review boards. All participants provided written informed consent.

\section{SMOKING ASSESSMENT}

Average number of daily cigarettes smoked during the week before treatment initiation, the week before the laboratory session, and the week before the 17-week follow-up were assessed at in-person visits by a research assistant using the Timeline Follow Back (TLFB) method (Sobell and Sobell, 1992). Selfreported abstinence was assessed using TLFB and verified by an exhaled carbon monoxide measurement of $\leq 10$ parts per million (Benowitz et al., 2002).

\section{LABORATORY PROCEDURE}

Within two weeks of treatment completion, subjects participated in a $1.5 \mathrm{~h}$ laboratory session that included practicing meditation while supine in an fMRI scanner. Participants were instructed in two standard mindfulness meditations: (1) Mindfulness of the breath ("Please pay attention to the physical sensation of the breath wherever you feel it most strongly in the body. Follow the natural and spontaneous movement of the breath, not trying to change it in any way. Just pay attention to it. If you find that your attention has wandered to something else, gently but firmly bring it back to the physical sensation of the breath."); and (2) Loving-kindness ("please think of a time when you genuinely wished someone well. Using this feeling as a focus, silently wish all beings well, by repeating a few short phrases of your choosing.") (Gunaratana, 2002). Subjects practiced each meditation before the laboratory session and confirmed that they could follow the instructions. Each run consisted of a 2 min baseline period ("please close your eyes and don't think of anything in particular”), a $30 \mathrm{~s}$ recorded meditation instruction (as above), and a 4.5 min meditation period. Subjects performed each meditation twice in a blocked random order (i.e., AABB). After each run, participants rated how well they were able to follow the instructions during each meditation period (0-10). After the last meditation, participants rated their level of anxiety (0-10).

\section{HRV ACQUISITION AND PROCESSING}

Heart rate was recorded using a pressure transducer attached to each participant's non-dominant thumb. This method has been previously shown to accurately capture interbeat variability that is comparable to standard Electrocardiography and was pilottested in our laboratory using simultaneous pulse transduction and ECG before proceeding (Heilman et al., 2008). A Biopac MP150 pressure transduction system running AcqKnowledge 4.1 software (Biopac Systems, USA), with a $1000 \mathrm{~Hz}$ sample rate and $1 \mathrm{~Hz}$ high band pass filter, was used for acquiring and processing heart rate data. Heart rate waveforms were analyzed to determine appropriateness for spectral analysis and deviant interbeat intervals were identified and edited by visual inspection according to current guidelines (Task Force, 1996). For heart rate data to be considered valid, the waveforms were required to have at least 120 consecutive seconds of identifiable HR data with no more than four consecutive, and no more than $20 \%$ total, interpolated beats. HF-HRV was defined as the power in the frequency range of $0.15 \mathrm{~Hz}$ to $0.4 \mathrm{~Hz}$ (Task Force, 1996). Spectral estimates of power were logarithmically transformed to normalize the distribution of scores. Two consecutive 2 min segments were derived from each of the meditation periods and averaged so that 
comparisons could be made with our two-minute baseline periods; two minute periods have been shown to provide sufficient data for the analysis of HRV (Task Force, 1996; Berntson et al., 1997). There were no differences in HF-HRV between the two types of meditation $(p=0.772)$, and thus they were combined into a single variable for analysis. HF-HRV values from baseline were subtracted from meditation values to derive an HF-HRV change score (change $=$ meditation - baseline $)$.

Respiration rate was recorded using a pressure transducer affixed to the lower thorax via an elastic band. The Biopac MP150 system running AcqKnowledge 4.1 software (Biopac Systems, USA), was used for acquiring and processing respiration rate data. Although there are differences of opinion regarding the need to account for respiration when quantifying parasympathetic output, HF-HRV uncorrected for respiration is accepted as a valid index of cardiac parasympathetic control when there are no task differences in respiration (Denver et al., 2007; Grossman and Taylor, 2007), and is a meaningful index of cardiorespiratory control that has predictive power independent of the underlying parasympathetic mechanisms (Ritz and Dahme, 2006; Egizio et al., 2011). In our sample, there were no differences in respiration rate between baseline and meditation periods $(t=-0.184$, $p=0.855)$ and thus HF-HRV data are presented without respiratory adjustment.

\section{STATISTICAL ANALYSIS}

Statistical analyses were performed in SPSS 18.0. Three outlying cases with HF-HRV data greater than three standard deviations below the mean were excluded before further analysis.

We constructed four linear regression models with average number of daily cigarettes smoked at week 17 as the outcome. The main predictor variables in models $1,2,3$, and 4 were baseline HF-HRV, direction of HF-HRV change (increase or decrease), HF-HRV during meditation, and magnitude of HF-HRV change, respectively. All models included age, gender, and smoking behavior both at pre- and post-treatment as a priori covariates because they are known to be associated with HF-HRV (Hayano et al., 1990; Britton et al., 2007). Treatment group was also added as a covariate in each of the models, and baseline HF-HRV was included as a covariate in models 2, 3, and 4 (Jennings and Gianaros, 2007). All tests are two-tailed; Bonferroni correction was used to control for Type I error associated with multiple comparisons; therefore, significance is reported as $p=0.0125$ (Rom, 1990).

\section{RESULTS \\ PARTICIPANT DEMOGRAPHICS}

Participants' demographic characteristics are presented in Table 1. Overall, $46 \%$ of participants were members of ethnic minority groups, and $64 \%$ were men. On average, participants were 48 years old, smoked 19 cigarettes per day, started smoking regularly at the age of 17 , and had 5.9 previous quit attempts.

\section{MANIPULATION CHECK}

Participants reported that they were able to follow instructions to a high degree (Mean $=7.3 \pm 2.2$ on a $0-10$ Likert scale). There was no relationship between participant ratings and baseline
Table 1 | Pre-treatment characteristics of participants.

\begin{tabular}{ll}
\hline & Participants ( $\mathbf{n} \mathbf{2 8})$ \\
\hline SEX & $N(\%)$ \\
Male & $18(64.3)$ \\
Female & $10(35.7)$ \\
AGE & $48.00(8.93)$ \\
RACE & \\
White & 15 \\
Black & 11 \\
Hispanic & 2 \\
EDUCATION LEVEL & \\
College grad or more & 17 \\
Partial college & 2 \\
High school & 9 \\
\hline & Mean (SD) \\
\hline Started smoking 3x/week (age) & 16.61 (4.25) \\
Number of cigarettes/day & $18.86(8.30)$ \\
Number of prior quit attempts & $5.86(7.78)$ \\
\hline
\end{tabular}

Table 2 | Mean HF-HRV values in normalized units (n.u.)

\begin{tabular}{lll}
\hline & Mean (SD) & Range \\
\hline Mean baseline HF-HRV & $6.477(0.257)$ & $5.747-7.018$ \\
Mean HF-HRV during meditation & $6.490(0.254)$ & $5.756-6.998$ \\
Mean HF-HRV change score & $0.0111(0.063)$ & $-0.170-0.1264$ \\
\hline
\end{tabular}

HF-HRV ( $r=0.022, p=0.912)$ or changes in HF-HRV during meditation $(r=-0.013, p=0.949)$. Participants reported mild-moderate anxiety in the scanner $(4.1 \pm 2.6)$.

\section{GROUP DIFFERENCES IN HF-HRV}

Independent-samples $t$-tests indicated that there were no differences between individuals who received mindfulness training and those who received Freedom From Smoking in baseline HF-HRV, HF-HRV during the meditations, or mean HF-HRV meditation reactivity, all p's $>0.05$.

\section{HF-HRV AS A PREDICTOR OF SMOKING OUTCOMES}

Table 2 presents the mean and range of average HF-HRV during baseline, meditation, and the magnitude of change from baseline to meditation. There were no significant differences in HF-HRV between baseline and meditation $\left(t_{(27)}=-0.954, p=0.349\right)$, indicating that on average, there was no acute change in HF-HRV. However, participants were significantly more likely to exhibit an increase in HF-HRV than a decrease in HF-HRV from resting baseline to meditation; Twenty of 28 participants (71.4\%) exhibited an average increase, and eight participants (28.6\%) exhibited an average decrease in HF-HRV from baseline to meditation $\left(\chi_{(1)}^{2}=5.14, p=0.023\right)$.

After adjustment for covariates, HF-HRV during baseline was not significantly associated with number of cigarettes smoked at week 17 ( $\left.b=5.028, \mathrm{SE}=3.783, \Delta r^{2}=0.035, p=0.198\right)$. 
The models predicting number of cigarettes smoked at week 17 from HF-HRV during meditation $(b=-27.016, \mathrm{SE}=13.494$, $\left.\Delta r^{2}=0.070, p=0.059\right)$, and from the magnitude of change in HF-HRV $\left(b=-30.555, \mathrm{SE}=13.916, \Delta r^{2}=0.082, p=\right.$ $0.040)$, approached, but did not reach statistical significance after correction for multiple comparisons. However, when change in HF-HRV was dichotomized by direction of change (increase vs. decrease), regression analyses showed that this significantly predicted the number of cigarettes smoked per day at week 17, independently of the effects of age, gender, treatment group, number of cigarettes smoked the week prior to treatment initiation, number of cigarettes smoked the week prior to the laboratory session, and baseline HF-HRV $\left(b=-4.89\right.$, SE $=1.65, \Delta r^{2}=0.128, p=$ $0.008)$. These results did not meaningfully change when anxiety ratings were added to the model. Twenty percent of participants who exhibited an increase in HF-HRV and $12.5 \%$ of participants who exhibited a decrease in HF-HRV achieved smoking abstinence at week 17, as verified by exhaled carbon monoxide measurement of $=10 \mathrm{ppm}$ (sample size insufficient for $\chi^{2}$ analysis).

\section{DISCUSSION}

In sum, one of our four hypotheses was definitively supported, two had moderate support and one was refuted. In the current study, individuals who demonstrated an increase in HFHRV from baseline to meditation smoked significantly fewer cigarettes 17-weeks after treatment initiation, compared to those who demonstrated a decrease in HF-HRV. The magnitude of this change and HF-HRV during meditation was each moderately associated with smoking outcomes, while baseline levels of HF-HRV were not predictive of smoking outcomes.

The differential autonomic responses to the meditation conditions may be reflective of the inter-individual variability in HF-HRV reactivity in response to meditation. Previous studies of both novice and experienced meditators have shown average increases in HF-HRV during meditation, although individual differences in the direction of change have been reported (Lehrer et al., 1999; Takahashi et al., 2005; Peressutti et al., 2010). Consistent with these reports, we found that $71 \%$ of participants showed increased HF-HRV. The decrease in HF-HRV among some participants may have been due to the mildly stressful environment of the laboratory. Importantly, an increase, compared to a decrease in HF-HRV was significantly associated with subsequent reduced cigarette consumption, while HF-HRV during baseline, during meditation, and the magnitude of change from baseline to meditation were not. This is consistent with the idea that direction of phasic HF-HRV change during a meditation exercise is associated with psychological health (Rockliff et al., 2008). Although preliminary, these results suggest that the ability to bring autonomic activity into a parasympathetically-dominant state while meditating may be a more significant predictor of substance use outcomes than tonic levels of autonomic activity or magnitude of change. As noted above, the magnitude of change in HF-HRV was marginally significantly associated with smoking outcomes. Future research should use larger samples to replicate these results and to examine whether magnitude of increase may also relate to smoking outcomes.
There were no differences in any of the HF-HRV measures between novices and individuals who received 4-weeks of mindfulness training. This is consistent with the notion that individuals can learn to meditate without long periods of training (Zeidan et al., 2010b; Moyer et al., 2011). This also suggests, however, that 4-weeks of practice may not be sufficient for some individuals to achieve the ability to drop into a parasympathetically-dominated psychophysiological state during meditation. As indicated by moderate levels of anxiety reported while in the fMRI scanner, this environment may be a sufficient provocateur for differentiating individuals who have this capacity from those who do not, regardless of previous mindfulness training. As such, HFHRV changes from baseline to meditation in a mildly stressful environment may be a potential biomarker or predictor for individuals who are more likely to be successful in quitting smoking. Given previous research suggesting that HF-HRV may index selfregulatory capacity, those individuals who exhibited increases in HF-HRV when meditating may have the self-control resources necessary to inhibit habitual smoking behaviors. If these results are replicated, future smoking cessation studies may be able to use similar, non-invasive measures to individualize target quit dates, waiting until meditation during mild stress results in a consistent increase in HF-HRV before committing to a quit date. Tailoring quit dates to match self-regulatory capacity may reduce relapses and minimize failure experiences resulting in improved smoking cessation outcomes.

There are several limitations of this study. First, it was conducted with a subsample of participants from a larger RCT. Therefore, we may have been underpowered to detect effects of HF-HRV during baseline and/or meditation on smoking outcomes. A larger sample size may be better suited to detect whether these predict smoking outcomes as well. Second, the reported association may not be specific to mindfulness meditation; other tasks that result in an acute increase in HF-HRV may produce similar results. Future research comparing the predictive power of acute HF-HRV increases in response to other meditative or relaxation tasks is warranted. In addition, future studies should include comparisons between the predictive power of stress-induced (Ashare et al., 2012) versus meditation-induced changes in HF-HRV. Third, the meditation laboratory session occurred after a 4-week smoking cessation study. Future studies that assess HF-HRV at both pre- and post-treatment may more precisely elucidate potential relationships between changes in parasympathetic output during treatment and subsequent outcomes. Finally, although our results are consistent with proposed mechanisms of mindfulness and prefrontal inhibition of habitual smoking behavior, we did not directly measure these constructs. The mechanisms through which HF-HRV changes from baseline to meditation may predict smoking outcomes might be further clarified by combining these measures with others, such as ecological momentary assessment of coping responses to stress and cravings.

In conclusion, this study demonstrated that meditation more often resulted in an acute increase in HF-HRV than a decrease in HF-HRV in a nicotine-dependent sample. Importantly, we showed for the first time that individuals exhibiting an increase in HF-HRV while meditating had better smoking outcomes three 
months later, compared to those who exhibited a decrease in HF-HRV. These findings, although preliminary, suggest that measurements of acute HF-HRV changes during meditation show promise as a non-invasive biomarker and predictor of smoking cessation treatment.

\section{ACKNOWLEDGMENTS}

We would first like to thank the participants in this study. Secondly, we'd like to thank Dr. Paul Grossman for consultation regarding the issue of respiration in HF-HRV assessment. Thirdly, we'd like to thank Dr. Rachel Lampert for her suggestions and advice on HF-HRV analysis. Fourthly, we'd like to thank Drs. Bruce Rounsaville, Kathleen Carroll, and Marc Potenza for their suggestions and advice on the clinical aspects of this study. Finally, we'd like to thank the staff of the Yale Therapeutic
Neuroscience Clinic, and Psychotherapy Development Research Center.

\section{FUNDING}

This study was funded by the following grants: NIDA K12DA00167, P50-DA09241, UL 1 DE019586-02, the US Veterans Affairs New England Mental Illness Research, Education, and Clinical Center (MIRECC), and the Office of Academic Affiliations, Advanced Fellowship Program in Mental Illness Research and Treatment, Department of Veterans Affairs. The NIDA and VA had no further role in study design; in the collection, analysis and interpretation of data; in the writing of the report; or in the decision to submit the paper for publication.

ClinicalTrials.gov identifier: NCT01144689.

\section{REFERENCES}

Allen, M. T., Matthews, K. A., and Kenyon, K. L. (2000). The relationships of resting baroreflex sensitivity, heart rate variability and measures of impulse control in children and adolescents. Int. J. Psychophysiol. 37, 185-194.

Appelhans, B. M., and Luecken, L. J. (2006). Heart rate variability as an index of regulated emotional responding. Rev. Gen. Psychol. 10, 229-240.

Arch, J. J., and Craske, M. G. (2006). Mechanisms of mindfulness: emotion regulation following a focused breathing induction. Behav. Res. Ther. 44, 1849-1858.

Ashare, R., Sinha, R., Lampert, R., Weinberger, A., Anderson, G., Lavery, M., Yanagisawa, K., and Mckee, S. (2012). Blunted vagal reactivity predicts stressprecipitated tobacco smoking. Psychopharmacology 220, 259-268.

Benowitz, N. L., Jacob Iii, P., Ahijevych, K., Jarvis, M. J., Hall, S., Lehouezec, J., Hansson, A., Lichtenstein, E., Henningfield, J., Tsoh, J., Hurt, R. D., and Velicer, W. (2002). Biochemical verification of tobacco use and cessation. Nicotine Tob. Res. 4, 149-159.

Berntson, G. G., Bigger, J. T. J., Eckberg, D. L., Grossman, P., Kaufmann, P. G., Malik, M., Nagaraja, H. N., Porges, S. W., Saul, J. P., Stone, P. H., and van der Molen, M. W. (1997). Heart rate variability: origins, methods, and interpretive caveats. Psychophysiology 34, 623-648.

Berntson, G. G., Cacioppo, J. T., Binkley, P. F., Uchino, B. N., Quigley, K. S., and Fieldstone, A. (1994). Autonomic cardiac control. III. Psychological stress and cardiac response in autonomic space as revealed by pharmacological blockades. Psychophysiology 31, 599-608.

Berntson, G. G., Cacioppo, J. T. and Quigley, K. S. (1993a). Cardiac psychophysiology and autonomic space in humans: empirical perspectives and conceptual implications. Psychol. Bull. 114, 296-322.

Berntson, G. G., Cacioppo, J. T., and Quigley, K. S. (1993b). Respiratory sinus arrhythmia: autonomic origins, physiological mechanisms, and psychophysiological implications. Psychophysiology 30, 183-196.

Bleil, M. E., Gianaros, P. J., Jennings, J. R., Flory, J. D., and Manuck, S. B. (2008). Trait negative affect: toward an integrated model of understanding psychological risk for impairment in cardiac autonomic function. Psychosom. Med. 70, 328-337.

Bowen, S., Chawla, N., Collins, S. E., Witkiewitz, K., Hsu, S., Grow, J., Clifasefi, S., Garner, M., Douglass, A., Larimer, M. E., and Marlatt, A. (2009). Mindfulness-based relapse prevention for substance use disorders: a pilot efficacy trial. Subst. Abus. 30, 295-305.

Bowen, S., and Marlatt, A. (2009). Surfing the urge: brief mindfulnessbased intervention for college student smokers. Psychol. Addict. Behav. 23, 666-671.

Brewer, J. A., Bowen, S., Smith, J. T., Marlatt, G. A., and Potenza, M. N. (2010). Mindfulness-based treatments for co-occurring depression and substance use disorders: what can we learn from the brain? Addiction 105, 1698-1706.

Brewer, J. A., Mallik, S., Babuscio, T. A. Nich, C., Johnson, H. E., Deleone, C. M., Minnix-Cotton, C. A., Byrn,
S. A., Kober, H., Weinstein, A., Carroll, K., and Rounsaville, B. J. (2011). Mindfulness training for smoking cessation: results from a randomized controlled trial. Drug Alcohol Depend. 119, 72-80.

Brewer, J. A., Sinha, R., Chen, J. A., Michalsen, R. N., Babuscio, T. A., Nich, C., Grier, A., Bergquist, K. L., Reis, D. L., Potenza, M. N., Carroll, K. M., and Rounsaville, B. J. (2009). Mindfulness training and stress reactivity in substance abuse: results from a randomized, controlled stage I pilot study. Subst. Abus. 30, 306-317.

Britton, A., Shipley, M., Malik, M., Hnatkova, K., Hemingway, H., and Marmot, M. (2007). Changes in heart rate and heart rate variability over time in middle-aged men and women in the general population. Am. J. Cardiol. 100, 524-527.

Broderick, P. (2005). Mindfulness and coping with dysphoric mood: contrasts with rumination and distraction. Cognit. Ther. Res. 29, 501-510.

Carney, R. M., Freedland, K. E., and Veith, R. C. (2005). Depression, the autonomic nervous system, and coronary heart disease. Psychosom. Med. 67, S29-S33.

Cohen, H., Kaplan, Z., Kotler, M., Mittelman, I., Osher, Y., and Bersudsky, Y. (2003). Impaired heart rate variability in euthymic bipolar patients. Bipolar Disord. 5, 138-143.

Cohen, H., Kotler, M., Matar, M. A., Kaplan, Z., Miodownik, H., and Cassuto, Y. (1997). Power spectral analysis of heart rate variability in posttraumatic stress disorder patients. Biol. Psychiatry 41, 627-629.
Cohen, H., Kotler, M., Matar, M. A., Kaplan, Z., Loewenthal, U., Miodownik, H., and Cassuto, Y. (1998). Analysis of heart rate variability in posttraumatic stress disorder patients in response to a trauma-related reminder. Biol. Psychiatry 44, 1054-1059.

Craske, M. G., Lang, A. J., Aikins, D., and Mystkowski, J. L. (2005). Cognitive behavioral therapy for nocturnal panic. Behav. Ther. 36, 43-54.

Cropley, M., Ussher, M., and Charitou, E. (2007). Acute effects of a guided relaxation routine (body scan) on tobacco withdrawal symptoms and cravings in abstinent smokers. Addiction 102, 989-993.

Davis, J., Fleming, M., Bonus, K., and Baker, T. (2007). A pilot study on mindfulness based stress reduction for smokers. BMC Complementary and Alternative Medicine 7, 2.

Demaree, H. A., Robinson, J. L., Everhart, D. E., and Schmeichel, B. J. (2002). Resting RSA is associated with natural and selfregulated responses to negative emotional stimuli. Brain Cogn 56, 14-23.

Denver, J. W., Reed, S. F., and Porges, S. W. (2007). Methodological issues in the quantification of respiratory sinus arrhythmia. Biol. Psychol. 74, 286-294.

Ditto, B., Eclache, M., and Goldman, N. (2006). Short-term autonomic and cardiovascular effects of mindfulness body scan meditation. Ann. Behav. Med. 32, 227-234.

Egizio, V. B., Eddy, M., Robinson, M., and Jennings, J. R. (2011). Efficient and cost-effective estimation of the influence of respiratory variables on respiratory sinus arrhythmia. Psychophysiology 48, 488-494. 
El-Sheikh, M. (2001). Parental drinking problems and children's adjustment: vagal regulation and emotional reactivity as pathways and moderators of risk. J. Abnorm. Psychol. 110, 499-515.

Fabes, R. A., and Eisenberg, N. (1997). Regulatory control and adults' stress-related responses to daily life events. J. Pers. Soc. Psychol. 73, 1107-1117.

Farb, N. A., Anderson, A. K., Mayberg, H., Bean, J., Mckeon, D., and Segal, Z. V. (2010). Minding one's emotions: mindfulness training alters the neural expression of sadness. Emotion 10, 25-33.

Flory, J. D., and Manuck, S. B. (2009). Impulsiveness and cigarette smoking. Psychosom. Med. 71, 431-437.

Friedman, B. H. (2007). An autonomic flexibility-neurovisceral integration model of anxiety and cardiac vagal tone. Biol. Psychol. 74, 185-199.

Friedman, B. H., and Thayer, J. F. (1998). Autonomic balance revisited: panic anxiety and heart rate variability. J. Psychosom. Res. 44, 133-151.

Fuller, B. F. (1992). The effects of stressanxiety and coping styles on heart rate variability. Int. J. Psychophysiol. $12,81-86$.

Gallagher, D., Terenzi, T., and De Meersman, R. (1992). Heart rate variability in smokers, sedentary and aerobically fit individuals. Clin. Auton. Res. 2, 383-387.

Goldin, P. R., and Gross, J. J. (2010). Effects of mindfulness-based stress reduction (MBSR) on emotion regulation in social anxiety disorder. Emotion 10, 83-91.

Grossman, P., and Taylor, E. W. (2007). Toward understanding respiratory sinus arrhythmia: relations to cardiac vagal tone, evolution and biobehavioral functions. Biol. Psychol. 74, 263-285.

Gunaratana, H. (2002). Mindfulness in Plain English. Somerville, MA: Wisdom Publications.

Hansen, A. L., Johnsen, B. H., Sollers, J. J., Stenvik, K., and Thayer, J. F. (2004). Heart rate variability and its relation to prefrontal cognitive function: the effects of training and detraining. Eur. J. Appl. Physiol. 93, 263-272.

Hayano, J., Yamada, M., Sakakibara, Y., Fujinami, T., Yokoyama, K., Watanabe, Y., and Takata, K. (1990). Short- and long-term effects of cigarette smoking on heart rate variability. Am. J. Cardiol. 65, 84-88.

Heilman, K., Handelman, M., Lewis, G., and Porges, S. (2008). Accuracy of the StressEraser in the detection of cardiac rhythms. Appl. Psychophysiol. Biofeedback 33, 83-89.

Jennings, J. R., and Gianaros, P. J. (2007). "Methodology," in Handbook of Psychophysiology, eds J. T. Cacioppo, L. G. Tassinary and G. G. Berntson (Cambridge, MA: Cambridge University Press). 812-833.

Johnsen, B. H., Thayer, J. F., Laberg, J. C., Wormnes, B., Raadal, M., Skaret, E., Kvale, G., and Berg, E. (2003). Attentional and physiological characteristics of patients with dental anxiety. J. Anxiety Disord. 17, 75-87.

Kok, B. E., and Fredrickson, B. L. (2010). Upward spirals of the heart: autonomic flexibility, as indexed by vagal tone, reciprocally and prospectively predicts positive emotions and social connectedness. Biol. Psychol. 85, 432-436.

Kupari, M., Virolainen, J., Koskinen, P., and Tikkanen, M. J. (1993). Shortterm heart rate variability and factors modifying the risk of coronary artery disease in a population sample. Am. J. Cardiol. 72, 897-903.

Lane, R. D., McRae, K., Reiman, E. M., Chen, K., Ahern, G. L., and Thayer, J. F. (2009). Neural correlates of heart rate variability during emotion. Neuroimage 44, 213-222.

Lehrer, P., Sasaki, Y., and Saito, Y. (1999). Zazen and cardiac variability. Psychosom. Med. 61, 812-821.

Light, K. C., Kothandapani, R., and Allen, M. T. (1998). Enhanced cardiovascular and catecholamine responses in women with depressive symptoms. Int. J. Psychophysiol. 28, 157-166.

McCraty, R., Atkinson, M., Tiller, W. A., Rein, G., and Watkins, A. D. (1995). The effects of emotions on short-term power spectrum analysis of heart rate variability. Am. J. Cardiol. 76, 1089-1093.

McDonald, A. H. (1980). "Mechanisms affecting heart-rate," in The Study of Heart Rate Variability, eds R. I. Kitney and O. Rompelman. (Oxford: Clarendon Press), 3-11.

Minami, J., Ishimitsu, T., and Matsuoka, H. (1999). Effects of smoking cessation on blood pressure and heart rate variability in habitual smokers. Hypertension 33 , 586-590.

Moore, A., and Malinowski, P. (2009). Meditation, mindfulness and cognitive flexibility. Conscious. Cogn. 18, 176-186.

Moyer, C. A., Donnelly, M. P. W., Anderson, J. C., Valek, K. C., Huckaby, S. J., Wiederholt, D. A.,
Doty, R. L., Rehlinger, A. S., and Rice, B. L. (2011). Frontal electroencephalographic asymmetry associated with positive emotion is produced by very brief meditation training. Psychol. Sci. 22, 1277-1279.

Nahshoni, E., Aravot, D., Aizenberg, D., Sigler, M., Zalsman, G., Strasberg, B., Imbar, S., Adler, E., and Weizman, A. (2004). Heart rate variability in patients with major depression. Psychosomatics 45, 129-134.

Oveis, C., Cohen, A. B., Gruber, J., Shiota, M. N., Haidt, J., and Keltner D. (2009). Resting respiratory sinus arrhythmia is associated with tonic positive emotionality. Emotion 9, 265-270.

Peressutti, C., Martín-González, J. M., García-Manso, J., and Mesa, D (2010). Heart rate dynamics in different levels of Zen meditation. Int. J. Cardiol. 145, 142-146.

Porges, S. W. (1996). Infant regulation of the vagal "brake" predicts child behavior problems: a psychobiological model of social behavior. Dev. Psychobiol. 29, 697.

Rechlin, T., Weis, M., Spitzer, A., and Kaschka, W. P. (1994). Are affective disorders associated with alterations of heart rate variability? J. Affect. Disord. 32, 271-275.

Ritz, T., and Dahme, B. (2006) Implementation and interpretation of respiratory sinus arrhythmia measures in psychosomatic medicine: practice against better evidence? Psychosom. Med. 68 617-627.

Rockliff, H., Gilbert, P., Mcewan, K., Lightman, S., and Glover, D. (2008). A pilot exploration of heart rate variability and salivary cortisol responses to compassion-focused imagery. Clin. Neuropsychiatry 5, 132-139.

Rom, D. M. (1990). A sequentially rejective test procedure based on a modified Bonferroni inequality. Biometrika 77, 663-665.

Rottenberg, J., Clift, A., Bolden, S., and Salomon, K. (2007). RSA fluctuation in major depressive disorder. Psychophysiology 44, 450-458.

Rottenberg, J., Salomon, K., Gross, J. J., and Gotlib, I. H. (2005). Vagal withdrawal to a sad film predicts subsequent recovery from depression. Psychophysiology 42, 277-281.

Rozanski, A., and Kubzansky, L. D. (2005). Psychologic functioning and physical health: a paradigm of flexibility. Psychosom. Med. 67, S47-S53.

Ruiz-Padial, E., Sollers, J. J. I., Vila, J., and Thayer, J. F. (2003). The rhythm of the heart in the blink of an eye: emotion-modulated startle magnitude covaries with heart rate variability. Psychophysiology 40, 306-313.

Sakakibara, M., and Takeuchi, S., and Hayano, J. (1994). Effect of relaxation training on cardiac parasympathetic tone. Psychophysiology 31, 223.

Sarang, P., and Telles, S. (2006). Effects of two yoga based relaxation techniques on heart rate variability (HRV). Int. J. Stress Manag. 13, 460-475.

Sayette, M. A. (2004). "Self-regulatory failure and addiction," in Handbook of Self-regulation: Research, Theory, and Applications, eds R. F. Baumeister and K. D. Vohs. (New York, NY: Guilford Press), 447-465.

Segerstrom, S. C., and Nes, L. S. (2007). Heart rate variability reflects self-regulatory strength, effort, and fatigue. Psychol. Sci. 18, 275-281.

Sloan, R. P., Shapiro, P. A., Bigger, T. J., Bagiella, E., Steinman, R. C. and Gorman, J. M. (1994). Cardiac autonomic control and hostility in healthy subjects. Am. J. Cardiol. 74, 298-300.

Sobell, L. C., and Sobell, M. B. (1992). "Timeline follow-back: a technique for assessing self-reported alcohol consumption," in Measuring Alcohol Consumption: Psychosocial and Biochemical Methods, eds R. Z. Litten and J. P. Allen. (Totowa, NJ: Humana Press). 41-50.

Takahashi, T., Murata, T., Hamada, T., Omori, M., Kosaka, H., Kikuchi, M. Yoshida, H., and Wada, Y. (2005). Changes in EEG and autonomic nervous activity during meditation and their association with personality traits. Int. J. Psychophysiol. 55, 199-207.

Task Force of the European Society of Cardiology, and the North American Society of Pacing and Electrophysiology. (1996). Heart rate variability: standards of measurement, physiological interpretation, and clinical use. Circulation 93, 1043-1065.

Taylor, V. A., Grant, J., Daneault, V., Scavone, G., Breton, E., Roffe-Vidal, S., Courtemanche, J., Lavarenne, A. S., and Beauregard, M. (2011). Impact of mindfulness on the neural responses to emotional pictures in experienced and beginner meditators. Neuroimage 57, 1524-1533.

Thayer, J. F., and Brosschot, J. F. (2005). Psychosomatics and psychopathology: looking up and down from the brain. Psychoneuroendocrinology 30 , 1050-1058. 
Thayer, J. F., Friedman, B. H., and Borkovec, T. D. (1996). Autonomic characteristics of generalized anxiety disorder and worry. Biol. Psychiatry 39, 255-266.

Thayer, J. F., Hansen, A., SausRose, E., and Johnsen, B. (2009). Heart rate variability, prefrontal neural function, and cognitive performance: the neurovisceral integration perspective on selfregulation, adaptation, and health. Ann. Behav. Med. 37, 141-153.

Thayer, J. F., and Lane, R. D. (2000). A model of neurovisceral integration in emotion regulation and dysregulation. J. Affect. Disord. 61, 201-216.

Weinberg, A., Klonsky, E. D., and Hajcak, G. (2009). Autonomic impairment in borderline personality disorder: a laboratory investigation. Brain Cogn. 71, 279-286.

Zeidan, F., Johnson, S. K., Diamond, B. J., David, Z., and Goolkasian, P. (2010a). Mindfulness meditation improves cognition: evidence of brief mental training. Conscious. Cogn. 19, 597-605.

Zeidan, F., Johnson, S. K., Gordon, N. S., and Goolkasian, P. (2010b).
Effects of brief and sham mindfulness meditation on mood and cardiovascular variables. $J$. Altern. Complement. Med. 16, 867-873.

Conflict of Interest Statement: The authors declare that the research was conducted in the absence of any commercial or financial relationships that could be construed as a potential conflict of interest.

Received: 30 November 2011; accepted: 29 February 2012; published online: 19 March 2012.
Citation: Libby DJ, Worhunsky $P D$, Pilver CE and Brewer JA (2012) Meditation-induced changes in highfrequency heart rate variability predict smoking outcomes. Front. Hum. Neurosci. 6:54. doi: 10.3389/fnhum. 2012.00054

Copyright (C) 2012 Libby, Worhunsky, Pilver and Brewer. This is an openaccess article distributed under the terms of the Creative Commons Attribution Non Commercial License, which permits non-commercial use, distribution, and reproduction in other forums, provided the original authors and source are credited. 